\title{
Collaborative Networks for Biodiversity Domain Organizations
}

\author{
Ekaterina Ermilova and Hamideh Afsarmanesh \\ University of Amsterdam, FCN group, \\ Science Park 107, 1098 XG Amsterdam, The Netherlands \\ \{e.ermilova, h.afsarmanesh\} @uva.nl
}

\begin{abstract}
European scientific research and development organizations, operating in the domains of biology, ecology, and biodiversity, strongly need to cooperate/collaborate with other centers. Unavailability of interoperation infrastructure as well as the needed collaboration environment among research organizations restricts their exchange of research results and efficient launching of co-working/co-development R\&D initiatives. This paper proposes the preestablishment of a long term strategic Collaborative Network called BTCN (Breeding environment for Temporary Collaborative Networks) for these organizations based on a survey of this environment. BTCN supports in a cost/time effective manner the formation and creation of potential future collaboration sub-networks addressing different $R \& D$ opportunities.
\end{abstract}

Keywords: Biodiversity, Collaborative Network, Breeding environment for Temporary Collaborative Networks.

\section{Introduction}

Organizations involved in biodiversity domain, e.g. data providers, research laboratories, universities, conservation groups, etc., referred to in this paper as BOs (Biodiversity-domain Organizations), are increasingly involved in collaborative activities with some other BOs as well as with some organizations outside the biodiversity domain, referred to here as EOs (External Organizations). For example, the collaboration scenario addressed in the FlySafe project [1], that is aimed at protection of airplanes from colliding with birds, identifies the co-working among several regional BOs involved in birds-related research, as well as a few EOs involved in weather forecast, airport administration, etc. Typically, such collaboration aims at supporting BOs with sharing their competencies and resources in order to together perform some monitoring, research, analysis, or development tasks at the level of a specific region, a country, or entire continent [2] [3]. The co-working and co-development approaches among BOs are increasingly growing in time as the proportion of complexity and demand of market / society opportunities in relation to existing resources and competencies and reaching beyond what single organizations can effectively support. Therefore, in order for BOs to increase their chances of involvement in emerging opportunities within the biodiversity domain, they need to 
get organized and supported by a special form of Collaborative Network (CN), which is referred to in this paper as the Breeding environment for Temporary Collaborative Networks (BTCN). The definition of BTCN is rooted in [4]:

\begin{abstract}
BTCN is a "strategic" alliance of Biodiversity Organizations (BOs), and External Organizations (EOs), which adhere to a base long term cooperation agreement, and adopt common operating principles and infrastructures, with the main goal of increasing the preparedness and their chances for participating in potential goal-oriented collaboration with others within Temporary Collaborative Networks (TCNs).
\end{abstract}

The main objective of the research addressed in this paper is both: (i) the study and characterization of existing forms of collaboration identified between European BOs, such as the networks of excellence and national research networks (as addressed in section 3), and (ii) the identification of new suitable forms of collaboration providing advanced support mechanisms to improve the efficiency of collaboration among the BOs (as addressed in section 4).

The research presented in this paper is partially performed within the FP7 project LifeWatch. This project aims at development of an e-Science \& Technology Infrastructure for Biodiversity Research, in which support of collaboration among biodiversity domain organizations is important task. More details about the research presented in this paper are addressed in a LifeWatch report [5].

\title{
2 Study of Related Research on Collaborative Networks
}

The research, presented in this paper, uses the main $R \& D$ results in the area of Collaborative Networks (CNs) as the primary input. Particularly, it applies: (1) the base concepts of Collaborative Networks, including the types and forms of CNs as well as the identified actors and their roles in long-term CNs, and (2) the ARCON modelling framework elements and the ARCON reference model for CNs. Below this section briefly addresses the $\mathrm{CN}$ classification and ARCON models.

The classification of CNs is addressed by the CN taxonomy introduced in [6]. In this taxonomy, all $\mathrm{CNs}$ are first divided into organized CNs that are called collaborative networked organizations, and the ad-hoc CNs. The collaborative networked organizations are further divided into two groups depending on their purpose and duration, namely divided into long-term strategic alliances and Goaloriented networks. The long-term strategic alliances typically exist for indefinite time and aim at preparation of their members for future collaboration activities. The goaloriented networks are created for a specific goal and exist until that goal is achieved. These networks are also further divided into two groups, by the type of their goals, namely into grasping-opportunity-driven-networks and continuous-activity-driven networks. The networks from the first group are formed on demand, when a new opportunity appears in the market or society. The networks from the second group have a longer term specific goal. Then, collaborative networked organizations are also divided by the types of their members, that can be either organizations or people. The long-term strategic alliances of organisation are called virtual organizations breeding environments (VBEs). Namely they represent breeding environments for 
formation and establishment the grasping-opportunity-driven-networks of organizations, which are called virtual organizations. The long-term strategic alliances of people are called professional virtual communities. In their turn they represent breeding environments for the grasping-opportunity-driven-networks of people, which are called virtual teams.

The ARCON (프 Reference model for COllaborative Networks) [7] is developed as an evolving system, constituting: (1) ARCON reference modeling framework, and (2) a set of ARCON reference models generated within this framework each representing one specific kind of $\mathrm{CN}$, for instance the virtual organizations breeding environments, virtual organizations, virtual teams, etc.

The ARCON framework comprehensively addresses the heterogeneous elements of different $\mathrm{CN}$ environments. In this model, the $\mathrm{CN}$ elements are grouped into two "subspaces" (as addressed in Fig. 1). The "CN Endogenous Elements" subspace represents all elements and components inside the $\mathrm{CN}$, while the " $C N$ Exogenous Interactions" subspace represents all elements and components outside the CN. Each subspace is divided into four different "dimensions" (i.e. the structural, componential, functional and behavioural dimensions for the "CN Endogenous Elements" subspace, and the market, support, societal and constituency dimensions for the "CN Exogenous Interactions" subspace). These two subspaces and their dimensions together represent the $\mathrm{CN}$ environment characteristics.

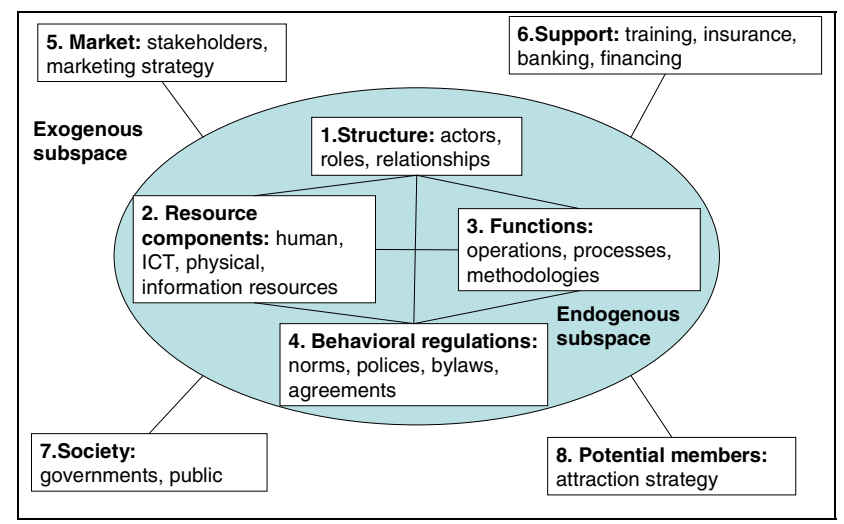

Fig. 1. Subspaces and dimensions in the ARCON reference framework

The above aspects of the $\mathrm{CN}$ are applied to the conduction of case studies and define the steps of our research on existing collaboration forms in the LifeWatch biodiversity domain (presented in section 3). Firstly, through applying the $\mathrm{CN}$ classification to our case study we have identified two specific types of CNs for which their 1st generation currently exist in the biodiversity domain. Secondly, using the ARCON reference model for CNs we have both: (1) characterised those existing $\mathrm{CNs}$, specifying their main elements (e.g. actors and their roles, resources, regulation documents, etc.), as well as (2) identified the requirements for their specific aspects, mechanisms, and tools that are needed to be supported for the LifeWatch CNs. 


\section{Case Study of Existing Collaboration Forms}

In order to study the current forms of collaboration among BOs, a questionnaire titled "Collaboration requirements of Biodiversity Organizations", is prepared and distributed among the biodiversity organizations participating in LifeWatch project.

The two main objectives of the study conducted through this questionnaire are addressed below:

Objective 1: Characterisation of the existing collaboration among different European BOs and other related EOs, as well as the identification of the current forms of these collaborations. To achieve this first objective, we specifically aimed at discovering whether the forms of collaboration, existing within the biodiversity domain, match one (or more) of the advanced forms currently defined CNs taxonomy, namely the CNO, long-term strategic alliance, goal-oriented network, virtual organization breeding environment, professional virtual community, virtual organization, or virtual team.

Objective 2: Identification of both the drawbacks/weaknesses of these current forms of collaboration, as well as the so far identified needs/requirements in these networks to improve the collaboration among BOs and other related EOs in Europe. To achieve this second objective, we were specifically aiming at understanding whether the European BOs have currently identified either some problems or some needs for improvements, related to current collaboration stage among different biodiversity centres, or the lack of it.

The participants at this case study represent more than ten major research organizations (BOs) from nine European countries involved in the LifeWatch consortium, including universities, large institutes, and research centres.

\subsection{Observations on Identified Collaboration Forms}

As addressed by the related research on Collaborative Networks, different forms of collaboration can be established among the organizations working in the biodiversity domain. All responders to the above questionnaire have indicated that they do regularly participate in some form of research related collaboration with other organizations, to enhance their effectiveness in their daily activities. This section further represents the main conclusions deduced related to different forms of existing biodiversity collaboration, involving European BOs. For better processing purposes, the main observations gathered through the questionnaire are categorised into the following five categories:

- Actors in collaboration. Currently the number of partners associated to a specific BO is directly proportional to the size of BO. Particularly, small BOs have far less chances to participate in cooperation and collaboration activities with others, and thus need more support for that. Also, currently while some organization types are strongly involved in collaboration with some types of BOs (e.g. data providers, research organizations, governmental organizations, etc.), there is a lack of collaboration with other organization types, such as organizations from industry, commercial/profit organizations, political organizations, and service (e.g. ICT-service) providers, while 
BOs express their interest in such collaborations. It means that current BOs are currently not benefiting from: (1) those opportunities that are associated with these organizations and (2) the support that these organizations can provide. For example, BOs currently lack the support that can be provided by ICT support providers. BOs are also lacking the opportunities to start new initiatives, e.g. an R\&D projects, involving commercial companies and industry.

- Existing forms of collaboration. There are currently two main forms of collaboration identified: goal-oriented networks that mainly represent Virtual Organizations with some elements of virtual teams. These alliances are named in this document as Temporary Collaborative Networks (TCNs). Long-term strategic alliances that mainly represent virtual organization breeding environments with some elements of professional virtual communities. These alliances are named in this document as Breeding Environment for Temporary Collaborative Networks (BTCNs). Other observations in this area are addressed in Table 1.

Table 1. Some observations about existing forms of collaboration

\begin{tabular}{l} 
Main reasons for existence of current long-term strategic alliances: They include: homogenization \\
of potential partners, accessing common infrastructure and assets, and funding new projects. \\
\hline Characterization of members in long-term strategic alliances and goal-oriented networks: \\
Currently, the partners of goal-oriented networks for the responders mainly overlap with their partners \\
in alliances, but there can be also some other external partners appearing for specific projects. It means \\
that responders state that current members of the long-term strategic alliances are not always enough for \\
addressing opportunities, namely they might not have enough possibilities to serve as breeding \\
environments for goal-oriented networks. The respondents to the questionnaire find it beneficial to \\
extend the alliances to larger networks of different organizations that can be potentially useful for \\
collaboration, so that they can search and select potential partners in these networks. \\
Early forms of long-term strategic alliances: Networks of excellence in biodiversity, such as ALTER- \\
net [8] and MARBEF [9] are referred to as early forms of long-term strategic alliances. \\
Main reasons for existence of current goal-oriented networks: They include: initiating and \\
performing projects and delivering assigned tasks, among others. \\
Main members' roles in long-term strategic alliances / goal-oriented networks: The specific roles that \\
members play in networks mainly represent the roles of regular actors and coordination/administration \\
actors. Some other roles are also weakly represented, such as Broker of new opportunities, Customer, \\
Support provider (e.g. training), Advisor or expert, Supplier, and Competitor. This means that the identified \\
CNs might lack the support role for BOs to improve themselves, for example training. Also, lacking the \\
role of Broker in long-term strategic alliances currently can result to absence of dynamism in finding and \\
addressing new opportunities.
\end{tabular}

- Collaboration opportunities. Currently, collaborative opportunities are understood by BOs as opportunities to collectively conduct research projects by a number of partners. Furthermore, these opportunities are typically brokered by scientific institutions and governments-related organizations. There is no mentioning of opportunities brokered by other organization types, e.g. commercial companies or industry. This is a serious limitation for current BOs activities and research results transfer and dissemination. Current long-term strategic alliances cannot address some emerged collaborative opportunities and form goal-oriented networks. Thus they need some support for becoming strong breeding environments for goal-oriented networks.

- Benefits of collaboration. The main benefit for collaboration among BOs is currently to pursue their "mission", namely performing research in the biodiversity area and achieving substantial result. Thus, this benefit constitutes the base for the 
BOs' value system. Consequently, measurement of research result achievement is the base for measurement of performance in biodiversity networks.

- Support for collaboration. Currently, to support remote collaboration, BOs use only basic ICT tools such as wiki, e-mail, web-sites. There is a lack of specific ICT tools supporting remote cooperation and collaboration (e.g. ontology management, performance measurement, etc.). Existing ICT tools represents only tools for storage, exchange and analysis of domain-related biodiversity data. Among the human resources, there are jobs (e.g. coordination, consultation, and technical jobs) that specially support collaboration. Among physical resources, there are recourses (e.g. ships and measurement devises) that are shared during collaboration activities.

\subsection{Analysis of Responses - Identified Requirements}

A main part of this case study also focused on investigating and identifying which support systems, tools, and functionalities are needed to be provided by long-term strategic alliances both to support their BO members as well as to support the administration of the networks. The supporting means and functionalities, as mainly addressed by their functional dimension of the ARCON reference model, are divided here into two groups, namely: (i) ICT support and background management activities and functionalities needed for long-term strategic alliances, and (ii) support tools and systems for dynamic creation of goal-oriented networks.

The conclusions in relation to these two groups are described below. For every support functionality, the percentage of responders who specified needing this functionality is also addressed below.

Main conclusions about requirements for ICT support and background management activities for long-term strategic alliances are addressed in Table 2:

Table 2. Requirements for ICT support and background activities for long-term strategic alliances

\footnotetext{
Common ICT infrastructure: Currently, the need for developing specialized ICT infrastructure to support cooperation and collaboration among the BOs is identified by $100 \%$ of responders. This is mainly maintained as required for cooperation among Biodiversity Organizations and with less necessity for supporting cooperation between the BOs and External Organizations such as governmentrelated organizations and public sector.

Governance system: Improving the governance system of current long-term strategic alliances is needed, as addressed by $70 \%$ of the responders.

Support-providers: Support-providing organizations are needed to be added within the long-term strategic alliances, as addressed by $55 \%$ of responders.

Bag of assets: A common "bag of assets" is needed to be established and properly managed by the long-term strategic alliances, as addressed by $100 \%$ of responders.

Competency management: Management of BO's competency is required by the long-term strategic alliances, as addressed by $75 \%$ of responders.

Trust management: Trust establishment among BOs and its management is strongly needed within the long-term strategic alliances, as addressed by $100 \%$ of responders.

Common understanding: Increasing the common understanding of the general concepts of long-term strategic alliances as well as of the biodiversity domain concepts is required by the members of longterm strategic alliances, as addressed by $85 \%$ of responders.
} 
Main conclusions about ICT requirements for the dynamic creation of goaloriented networks within their breeding environments are addressed in Table 3.

Table 3. ICT requirements for the dynamic creation of goal-oriented networks

\begin{abstract}
Need to support dynamic creation of goal-oriented networks: BOs do not have so far enough experience and/or knowledge about the $\mathrm{CN}$ and the possibilities for dynamic creation of goal-oriented networks within long-term strategic alliances. Therefore they currently create goal-oriented networks mainly in the traditional way. Namely, from the open universe of organizations and individuals, but primarily with those BOs with which they have past experiences. The support for dynamic creation of goal-oriented networks is stated to be needed by all types of national and international partner organizations, except for forming a new goal-oriented network with BOs that have a long history of collaboration in the past.

Agility in creation: BOs would like to see an increase in agility of creation of goal-oriented networks, as indicated by the responders.

Size of goal-oriented networks: BOs also find it useful to support acquiring a larger size (through formation of goal-oriented networks) responding to bids for most projects.

Chances for involvement: BOs indicated the need to increase their chances for involvement into goaloriented networks.

Creation risks for goal-oriented networks: BOs indicated the need for, to the extent possible, decreasing the potential of risks in creation of goal-oriented networks especially for larger networks.
\end{abstract}

\title{
3.3 Conclusions on Restrictions for Existing Cooperation/Collaboration among BOs
}

The main conclusions reached from our case study are summarised below.

1_ Lack of homogeneity in chances for BOs. The responders have indicated that there is no uniformity between different BOs, which in turn strongly affects their chances for collaboration. Namely the identified heterogeneity includes:

- Size of BOs (that ranges from 5 to 300 employees) that usually proportionally affects their resource capacity (e.g. amount of human or machinery recourses) as well as their lists of contacts/partners. Namely, small organizations do not have much recourses and contacts with other organizations. It means that even if small BOs are competent in performing some specific tasks, since they do not have enough resource capacities, they have difficulties for involvement into collaboration with others. Similarly, if they have a small number of contacts, currently it is difficult to get involved in TCN partnership that gets established to address an emerged opportunity.

- Locality of partners (that ranges from dominated by local partners to wide variety of local and international partners) that also affects their chances of collaboration involvement. Organizations which (due to some reasons, e.g. tradition of the geographic location) can only collaborate nationally, miss those benefits that can be provided by international collaboration.

- Range of partner types (that ranges from partners in a similar area of activity to partners from multiple areas of activity) affects the capabilities within the collaboration projects. For example, the BOs who collaborate only with those BOs which have the same area of activity (e.g. marine biology) do not have opportunity to address collaborative opportunities requiring a merge of capabilities.

- Age of organizations (that ranges from decades to centuries) that directly affects the number of currently established relationships with other organizations. Older 
organizations have longer and larger partnerships with others, thus for them it is easier to find trustworthy partners to address collaborative opportunities.

- Expertise in the area of CNs (that ranges from medium to high) that affects the smoothness of both the collaboration as well as the adoption of new $\mathrm{CN}$ approaches for biodiversity domain. For example, the organization that have a high expertise in the area of CNs are able to adopt new developed $\mathrm{CN}$ related approaches, and be a part of more advanced collaborative consortiums.

Therefore, some approaches and mechanisms developed within the $\mathrm{CN}$ discipline shall be applied to homogenize all BOs representation within BTCNs, homogenizing their chances for involvement into collaboration with others in potential TCNs.

2_ Limitation of fulfilling possibilities and lack of dynamism in creation of TCNs. The responders have indicated existence of a number of goal-oriented networks for biodiversity domain that constitute: a number of BOs, their related EOs, and a number of individual experts working together on a specific project. Earlier on, we introduced Temporary Collaborative Networks (TCN) referring to this kind of goal-oriented networks for the LifeWatch collaboration infrastructure. Current TCNs mainly represent projects aimed at specific research opportunities. The partners of TCNs are often selected among those which had some collaboration or cooperation experience in the past. However there are a large number of BOs that are at disadvantage, since they are not well-connected through any long-term strategic relationships. Furthermore, the current set of the Collaboration Networks are set statically, consequently posing a great obstacle for creation of time/cost effective and competitive dynamic TCN that require the pre-existence of a large strategic-alliance. The responses to questions about the requirements for dynamic TCN creation have indicated that BOs do not form TCNs dynamically, but rather traditionally, on the "friends of friends" basis, and therefore for this approach almost $40 \%$ of the responders indicated that currently there is no use for dynamic TCNs creation. However dynamism in TCN creation facilitates addressing more opportunities that are missed today due to the limitation of negotiation power, capabilities, etc. that are symptomatic of the existing closed long-term strategic alliances. Facilitating dynamic TCN creation shall therefore be studied and supported for future LifeWatch collaboration infrastructures.

3_ Small or loosely administrated 1st generation BTCNs. Some early forms of breeding environment for TCNs (BTCN) have been identified through the study. Specifically two types of BTCNs are identified through our study. First type of BTCNs represents alliances of partners with long-term and well established relationships. Typically they include national partners or some organizations of the same type. Currently, most such BTCNs are small, and thus they are not always of a large enough size to address collaborative opportunities only through their members, and therefore loose those opportunities. The second type of BTCNs includes a set of partners, who had some experience of both working together within past TCNs or past bilateral cooperation through which they have got to know each other. Examples of such BTCNs include networks of excellence in biodiversity. These BTCNs are typically large but the relationships between their members are loose and they lack the needed facilitating management functionalities of long-term strategic alliances. Consequently, these provide obstacles for effective joining of BOs competencies and for dynamically addressing collaborative opportunities. New generation of BTCNs 
should be established to support both better cooperation among a variety of members and better management of the BTCN, increasing possibilities of dynamic creation of TCNs.

4_ Lack of ICT collaboration support. While there ICT is currently used to support research in TCNs, e.g. many software tools, databases, etc., there is a lack of specialised ICT to support biodiversity collaboration infrastructure, e.g. negotiation support, partners' profiles and competencies management, common bag of assets management, trust management, etc.

5_ Heterogeneity of requirements to improve collaboration. Every responder identifies certain requirements as the "main" current obstacle in its way to effective collaboration, which is mostly unique and very different than other responses, as it represents the typical wide range of complexity to be addressed by the $\mathrm{CN}$ paradigm. These requirements depending on the $\mathrm{BO}$ and its location, widely varies and may range from the need to increase the number of partners with which to collaborate to the provision of lacking ICT support, or even establishing trust. While this observation indicates that there is no homogeneity between European BOs in relation to their situation and requirements for collaboration, on average more than $70 \%$ of responders have stated that they find the $\mathrm{CN}$-related support "very useful" for their collaboration activities.

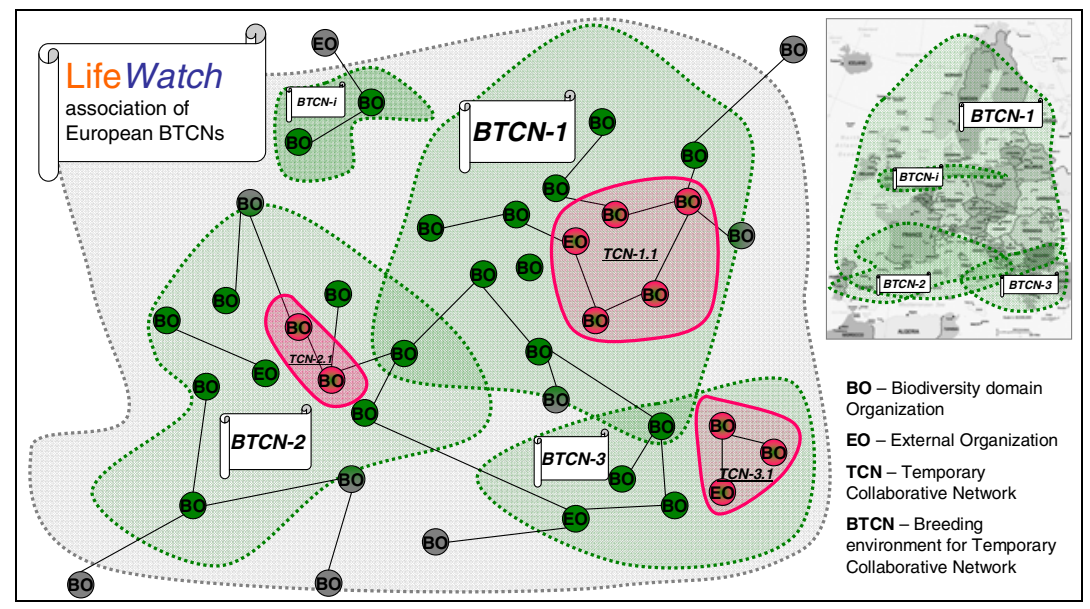

Fig. 2. LifeWatch association of European BTCNs

\section{Suggested Approach}

To address all requirements addressed in the previous sections, the new generation of BTCNs for this domain should be modelled, specified, and established. The $2^{\text {nd }}$ generation BTCNs shall strengthen relationships among their members that will support both: better cooperation among the members as well as better conditions for dynamic creation of TCNs. For the biodiversity domain, the BTCNs can be established either based on their geographical location, or based on their specialization area (e.g. 
for the marine domain, flora domain, weather domain under the aegis of the LifeWatch project, thus constructing the: "LifeWatch's association of European BTCNs", as illustrated in Fig. 2.

Furthermore, there are a number of questions that needs to be answered before specification and establishment of the $2^{\text {nd }}$ generation BTCNs, e.g.: What are the specific principles for clustering of BOs, EOs, and individual experts within BTCNs (BTCN typology)? How to attract BOs to join BTCNs and keep them interested and active? How should BTCNs be instantiated, organized, and coordinated? These questions will be further addressed in forthcoming papers.

\section{Conclusions}

This paper aims at addressing and applying the new developments and experiences achieved in the area of Collaborative Networks as potential solutions for establishing an effective collaboration infrastructure and for the European network of biodiversity research.

To achieve its aim, the paper presents: (i) study of the existing collaboration forms among European Biodiversity Organizations (BOs), (ii) identification of their types and complexities according to the existing $\mathrm{CN}$ 's taxonomy and reference model, (iii) identification of main requirements for improvement of BOs' collaboration, and (iv) suggestion of an effective and most suitable collaboration form for BOs' networks. The paper also suggests establishment of a long-term strategic network of BOs end their related external (non-biodiversity) organizations, which is called the BTCN (Breeding environment for Temporary Collaborative Networks).

\section{References}

1. FlySafe FP6 project, http: / /www.eu-flysafe.org/Project.html

2. Westley, F., Vredenburg, H.: Interorganizational Collaboration and the Preservation of Global Biodiversity. Organization Science 8(4), 381-403 (1997)

3. Guralnick, R.P., Hill, A.W., Lane, M.: Towards a collaborative, global infrastructure for biodiversity assessment. Ecology Letters 10(8), 663-672 (2007)

4. Afsarmanesh, H., Camarinha-Matos, L.M.: A framework for management of virtual organizations breeding environments. In: Collaborative networks and their breeding environments, pp. 35-48. Springer, Heidelberg (2005)

5. Ermilova, E., Afsarmanesh, A., Msanjila, M.: D5.2.a - Virtual Collaborative networks supporting LifeWatch R\&D infrastructure in biodiversity domain. Lifewatch (2009)

6. Camarinha-Matos, L.M., Afsarmanesh, H.: Collaboration forms. In: Collaborative networks reference modeling, pp. 51-66. Springer, New York (2008) ISBN: 978-0-387-79425-9

7. Camarinha-Matos, L.M., Afsarmanesh, H.: The ARCON modeling framework. In: Collaborative networks reference modeling, pp. 67-82. Springer, New York (2008) ISBN: 978-0-387-79425-9

8. ALTER-net - A Long-Term Biodiversity, Ecosystem and Awareness Research Network, http://www.alter-net.info

9. MARBEF - Marine Biodiversity and Ecosystem Functioning EU Network of Excellence, http: / / www . marbef . org 